\title{
Acousto-optic interaction enhancement in dual photonic-phononic cavities
}

\author{
N. Papanikolaou ${ }^{a}$, G. Gantzounis ${ }^{b}$, E. Almpanis $^{a}$, N. Stefanou ${ }^{c}$ \\ ${ }^{a}$ Institute of Microelectronics, NCSR "Demokritos", Ag. Paraskevi, GR-153 10 Athens, Greece \\ ${ }^{b}$ Graduate Aeronautical Laboratories (GALCIT) and Department of Applied Physics, \\ California Institute of Technology, Pasadena, California 911 25, USA \\ ${ }^{c}$ Section of Solid State Physics, University of Athens, Panepistimioupolis, GR-157 84, Athens, \\ Greece
}

\begin{abstract}
Light control through elastic waves is a well established and mature technology. The underlying mechanism is the scattering of light due to the dynamic modulation of the refractive index and the material interfaces caused by an elastic wave, the so-called acousto-optic interaction. This interaction can be enhanced in appropriately designed structures that simultaneously localize light and elastic waves in the same region of space and operate as dual optical-elastic cavities, often called phoxonic or optomechanical cavities. Typical examples of phoxonic cavities are multilayer films with a dielectric sandwiched between two Bragg mirrors or, in general, defects in macroscopically periodic structures that exhibit dual band gaps for light and elastic waves. In the present work we consider dielectric particles as phoxonic cavities and study the influence of elastic eigenmode vibrations on the optical Mie resonances. An important issue is the excitation of elastic waves in such submicron particles and, in this respect, we analyze the excitation of high-frequency vibrations following thermal expansion induced by the absorption of a femtosecond laser pulse. For spherical particles, homogeneous thermalization leads to excitation of the particle breathing modes. We report a thorough study of the acousto-optic interaction, correct to all orders in the acousto-optic coupling parameter, by means of rigorous full electrodynamic and elastodynamic calculations, in both time and frequency domains. Our results show that, under double elastic-optical resonance conditions, strong acousto-optic interaction takes place and results in large dynamical shifts of the high-Q optical Mie resonances, manifested through multiphonon exchange mechanisms.
\end{abstract}

Keywords: acousto-optic interaction, Mie resonances.

Further author information: (Send correspondence to N.P.)

N.P.: E-mail: N.Papanikolaou@imel.demokritos.gr, Telephone: +30 2106503245

Photonic Crystal Materials and Devices X, edited by Hernán Ruy Míguez, Sergei G. Romanov,

Lucio Claudio Andreani, Christian Seassal, Proc. of SPIE Vol. 8425, 84250M · @ 2012 SPIE

CCC code: $0277-786 \mathrm{X} / 12 / \$ 18 \cdot$ doi: $10.1117 / 12.922330$

Proc. of SPIE Vol. $842584250 \mathrm{M}-1$ 


\section{INTRODUCTION}

The synergy of light and sound waves in nanostructures opens new perspectives for novel functionalities in optical circuits and might enable the development of new concepts where phonons are used in storage and transmission of information in submicron devices. ${ }^{1}$ Recently there has also been intensive research on the socalled optomechanical interaction in cavities ${ }^{2}$ where mechanical motion is coupled to strongly localized light in a photonic cavity due to the radiation pressure. The light-sound coupling due to the acousto-optic (AO) interaction can be in principle enhanced if we achieve co-localization of elastic and optical energy. Cavities that simultaneously localize light and sound are sometimes termed phoxonic cavities and there exist few proposals for their realization in one dimensional (1D) multilayers ${ }^{3,4}$ as well as photonic crystal slabs. ${ }^{5-7}$ Photonic micro- $^{-}$ electromechanical systems vibrating at tens of $\mathrm{GHz}$ have been recently reported, ${ }^{8,9}$ while mechanical whispering gallery modes (WGM) were excited through optical WGM in $100 \mu \mathrm{m}$ silica spheres. The excitation was achieved by stimulated Brillouin scattering (SBS) where a pump signal triggered the mechanical vibration through a self-consistent process in which the two optical modes beat and, via the electrostrictive effect, apply stress to the material which drives the mechanical mode. ${ }^{9}$ Apart from silica, chalcogenide glasses are also investigated as candidates for photonic devices due to their large refractive index, low absorption, large nonlinear refractive index and high $\mathrm{AO}$ coefficient. High-Q $\mathrm{As}_{2} \mathrm{Se}_{3}$ microspheres were recently fabricated and their potential for high Q micoresonators was studied. ${ }^{10}$ Similarly, Brillouin lasing in a $\mathrm{CaF}_{2}$ WGM resonator has been reported ${ }^{11}$ using SBS which can be manipulated (suppressed or enhanced) by modifying the modal structure of the optical resonator. Simultaneous localization of light and sound can enhance the nonlinear effects and reduce the optical power required to trigger them. For smaller, nm-sized particles, ultrafast acoustic methods also exploit the $\mathrm{AO}$ interaction to study elastic wave propagation in nanostructures, which is monitored through changes in

the optical transmission or refrection. ${ }^{12-16}$ Alternative to pump-probe ultrafast acoustic methods, Brillouin or Raman inelastic light scattering (ILS) is also used to study the elastic response of nanoparticles. ${ }^{17}$ Here we will study AO interaction effects in micron-sized dielectric spherical particles.

\section{RESULTS AND DISCUSSION}

Simultaneous localization of light and sound can be achieved by defects in periodic structures. This direction was pursuited in recent studies of $1 \mathrm{D}$ multilayers and photonic crystal slabs. ${ }^{4,5}$ Light localization is possible also in single dielectric particles. It becomes more efficient for high-refractive-index materials and is manifested through the presence of the characteristic Mie resonances in the scattering cross section spectrum. Moreover, for an isolated particle in air, due to the large elastic impedance mismatch, the elastic eigenmodes have little coupling to air. For micron-sized particles we expect elastic vibrational eigenmodes with frequencies in the $\mathrm{GHz}$ 
range and it is important to note that the elastic response depends on the nature of excitation. The picosecond ultrasonics technique uses femtosecond laser pulses to generate elastic waves due to thermal expansion. We will consider an isotropic elastic excitation of a spherical particle, which means that we limit our discussion to radially symmetric elastic eigenmodes of the particle. Such an isotropic excitation can be achieved by thermal expansion following a femtosecond laser pulse that heats up the particle and triggers radial mechanical vibrations. The radially symmetric displacement field $\mathbf{U}(\mathbf{r}, t)=U(r, t) \hat{\mathbf{r}}$ is given by

$$
\frac{1}{c_{l}^{2}} \frac{\partial^{2} U(r, t)}{\partial t^{2}}=\frac{\partial^{2} U(r, t)}{\partial r^{2}}+\frac{2}{r} \frac{\partial U(r, t)}{\partial r}-2 \frac{U(r, t)}{r^{2}} .
$$

For a time harmonic solution of Eq. (1)

$$
U(r, t)=\Re[u(r) \exp (-i \Omega t)]
$$

the time independent equation satisfied by $u(r)$ is, for a free particle, a Sturm-Liouville problem, with appropriate boundary condidions, ${ }^{18}$ and the corresponding eigenfunctions are: $u_{n}(r)=j_{1}\left(\Omega_{n} r / c_{l}\right), n=0,1, \ldots$ where $j_{1}(x)$ is the spherical Bessel function of the first kind and order one. The eigenfunctions associated to eigenfrequencies $\Omega_{n}$ form an orthonormal basis set of acoustic breathing modes, which can be used to expand any radial displacement field. Dissipative and radiative losses can be included by assuming frequency-dependent complex longitudinal $c_{l}(1-i \alpha f)$ and transverse $c_{t}(1-i \alpha f)$ sound velocities with $f=\Omega / 2 \pi$ and a typical value for $\alpha=10^{-4} \mathrm{GHz}^{-1}$.

For a spherical particle, the field is expanded into spherical waves. ${ }^{19}$ An incident plane wave is expanded in this basis with appropriate coefficients $a_{P l m}^{0}$, where $P=E, H$ denote electric or magnetic polarization and $l m$ are angular momentum indices. The field around the particle is a sum of the incoming plane wave plus the scattered field, which can also be expanded into spherical waves with coefficients $a_{P l m}^{+}$. The latter are obtained by $a_{P l m}^{+}=T_{P l} a_{P l m}^{0}$, where the scattering T-matrix is numerically calculated through a recursive method for spherical multishell particles. ${ }^{20}$ When the AO interaction is present, the refractive index varies slowly compared to the period of the electromagnetic wave. Therefore the coefficients $a_{P l m}^{+}$oscillate also periodically with the angular frequency of the elastic wave and can be expanded into a Fourier series:

$$
a_{P l m}^{+}(t)=\sum_{n=0, \pm 1, \pm 2, \ldots} \tilde{a}_{P l m}^{+}(n) \exp \left(-i n \Omega_{0} t\right)
$$

The optical scattering cross section of the particle is defined through the expansion coefficients of the scattered field $a_{P l m}^{+}$in a spherical wave expansion

$$
\sigma_{s c}(t)=\frac{1}{q^{2}\left|E_{0}^{2}\right|} \sum_{P l m}\left|a_{P l m}^{+}(t)\right|^{2},
$$

where $q$ is the wavenumber and $E_{0}$ the amplitude of the incident plane wave. We are interested in the time variation of the optical scattering cross section during the elastic vibration launched due to heating by a femtosecond 

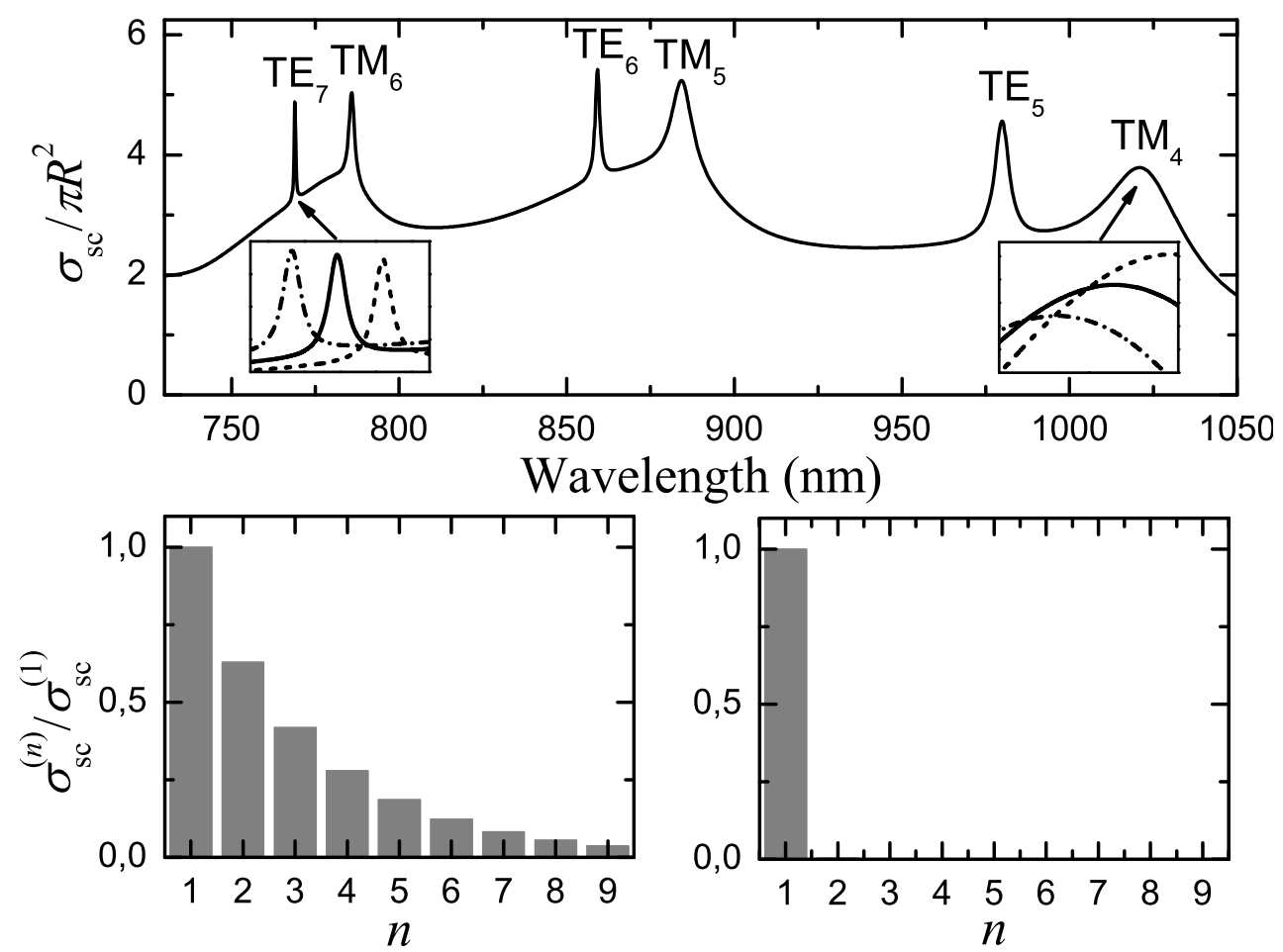

Figure 1. Upper diagram: Optical scattering cross section of a $\mathrm{As}_{2} \mathrm{Se}_{3}$ particle with radius $R=500 \mathrm{~nm}$. The insets show the variation of two Mie resonances $\left(\mathrm{TE}_{7}, \mathrm{TM}_{4}\right)$ upon excitation of the fundamental elastic breathing mode of the particle in a frequency window of $5 \mathrm{~nm}$. The snapshots are chosen to show the maximum variation corresponding to an initial displacement level $u_{0}=0.1 \mathrm{~nm}$. Lower diagrams: Partial cross sections associated with the scattered field [see Eq. (4),(6)] for the two Mie resonances: $\mathrm{TE}_{7}$ (left) and $\mathrm{TM}_{4}$ (right).

laser pulse. The elastic excitation causes a variation of the scattering spectra, because of the modification of the particle radius as well as the modulation of the refractive index which depends on the AO coefficient. Assuming an isotropic $\mathrm{AO}$ coefficient $\bar{p}$ and radially symmetric elastic excitation, the change in the refractive index is radially symmetric, depending on the elastic strain:

$$
\Delta n_{r}(r, t)=-\frac{1}{2} n_{r}^{3} \bar{p}\left[\frac{\partial U(r, t)}{\partial r}+2 \frac{U(r, t)}{r}\right]
$$

where $n_{r}$ the refractive index of the unperturbed particle.

For an amorphous $\mathrm{As}_{2} \mathrm{Se}_{3}$ particle of radius $R=500 \mathrm{~nm}$ radius, with density $\rho=3.08 \mathrm{gr} / \mathrm{cm}^{3}$ and longitudinal and transverse sound velocities $c_{l}=2685 \mathrm{~m} / \mathrm{s}$ and $c_{t}=1436 \mathrm{~m} / \mathrm{s}$, the first two breathing eigenmodes are at $f_{0}=2.282 \mathrm{GHz}$ and $f_{1}=5.206 \mathrm{GHz}$ with corresponding lifetimes $\tau_{0}=273 \mathrm{~ns}$ and $\tau_{1}=58$ ns. The fundamental breathing mode is predominantly excited following a homogeneous thermal expansion of the particle. Moreover, the lifetime of this mode is much longer than of higher order modes, so we can assume that we excite only the 
fundamental breathing mode and neglect losses. In this case a particle periodically vibrates with the frequency $f_{0}$. The optical response of the glass particle, calculated using the experimental refractive index ${ }^{21}$ in the near infrared exhibits a series of Mie resonances, which are transverse magnetic (TM) or transverse electric (TE), as shown in Fig. 1. The temporal variation of the optical spectra was calculated by taking snapshots of the vibrating particle and dividing the $\mathrm{As}_{2} \mathrm{Se}_{3}$ particle in a large number of subshells assuming a constant refractive index in each subshell calculated through Eq. (5) with $\bar{p}=0.25$. In practice, 40 subshells are sufficient to obtain convergence. The maximum displacement of the optical spectra is proportional to the intensity of the elastic field in the particle. This is clear also from the comparison of the spectral shifts for the high-Q $\left(\mathrm{TE}_{7}\right)$ and the low-Q $\left(\mathrm{TM}_{4}\right)$ resonanses shown in the insets of Fig. 1. However the strength of the AO interaction depends on the degree of localization of both optical and elastic fields as well as the overlap of their modal shapes. Apart from the temporal variation of the spectra we can calculate the ILS spectrum by averaging the scattering cross section over a period of the acoustic wave. Using Eq. (3) we can decompose the scattering cross section in Fourier components:

$$
\left\langle\sigma_{s c}\right\rangle=\frac{f}{q^{2}\left|E_{0}\right|^{2}} \sum_{P l m} \int_{0}^{1 / f} d t\left|a_{P l m}^{+}(t)\right|^{2}=\sum_{n} \frac{1}{q^{2}\left|E_{0}\right|^{2}} \sum_{P l m}\left|\tilde{a}_{P l m}^{+}(n)\right|^{2}=\sum_{n} \sigma_{s c}^{(n)},
$$

where $\sigma_{s c}^{(n)}$ is connected with the n-th order ILS process. The ILS spectrum for two characteristic Mie resonances, which is connected to the Fourier components of the scattered field, is shown in the lower part of Fig. 1. For the high-Q Mie resonance $\left(\mathrm{TE}_{7}\right)$, the Fourier spectrum has several components, indicating multiphonon absorptionemission processes. ${ }^{4,18}$ On the contrary for the broad, low-Q Mie resonance $\left(\mathrm{TM}_{4}\right)$, only the first-order Fourier component appears, and single phonon processes dominate. This latter case is the usual behavior expected in ILS experiments. It is important to note that the change in the optical spectra is due to the bulk AO effect which depends on the material AO coefficient.

The fundamental breathing mode has the longest lifetime, however for shorter times additional modes are also neaded to describe the particle vibration. The basic conclusions of our analysis hold even if we consider the excitation of higher-order breathing modes. In that case the vibration of the particle is not periodic, and the time dependence of the optical scattering cross section becomes complicated. The discrete Fourier transform [Eq. (3)] has to be generalized but the conclusions for the temporal variation of the optical cross section and the anaysis on the influence of the AO interaction strenght on the ILS spectra is still valid. ${ }^{18}$

\section{CONCLUSION}

In summary, we have studied the optical and elastic properties of amorphous $\mathrm{As}_{2} \mathrm{~S}_{3}$ micron-sized spherical particles under excitation of elastic waves generated by a femtosecond laser pulse. Strong AO interaction can be 
expected in the dielectric particle, which will be manifested by the occurrence of multiphonon exchange processes and their signature in the ILS spectra.

\section{ACKNOWLEDGMENTS}

This work is supported by the European Commission Seventh Framework Program (FP7) under the FET-Open project TAILPHOX N 233833.

\section{REFERENCES}

1. T. Gorishnyy, M. Maldovan, C. Ullah, and E. L. Thomas, Physics World 18, 24 (2005).

2. M. Eichenfield, J. Chan, R. M. Camacho, K. J. Vahala, and O. Painter, Nature (London) 462, 78 (2009).

3. P. Lacharmoise, A. Fainstein, B. Jusserand, V. Thierry-Mieg Appl. Phys. Lett. 84, 3274 (2004).

4. I. E. Psarobas, N. Papanikolaou, N. Stefanou, B. Djafari-Rouhani, B. Bonello, V. Laude, Phys. Rev. B 82, $174303(2010)$.

5. V. Laude, J.-C. Beugnot, S. Benchabane, Y. Pennec, B. Djafari-Rouhani, N. Papanikolaou, J. M. Escalante, and A. Martinez Opt. Exp. 19, 9690 (2011).

6. D. A. Fuhrmann, S. M. Thon, H. Kim, D. Bouwmeester, P. M. Petroff, A. Wixforth, H. J. Krenner, Nature Photonics 5, 605 (2011).

7. J. Chan, T. P. Mayer Alegre, A. H. Safavi-Naeini, J. T. Hill, A. Krause, S. Gröblacher, M. Aspelmeyer, O. Painter, Nature 478, 89 (2011).

8. T. Carmon, K. J. Vahala, Phys. Rev. Lett. 98, 123901 (2007).

9. M. Tomes, T. Karmon, Phys. Rev. Lett. 102, 113601 (2009).

10. D. H. Broaddus, M. A. Foster, I. H. Agha, J. T. Robinson, M. Lipson, A. L. Gaeta, Opt. Exp. 17, 5998 (2009).

11. I. S. Grudinin, A. B. Matsko, L. Maleki, Phys. Rev. Lett. 102, 043902 (2009).

12. V. Juvé, A. Crut, P. Maioli, M. Pellarin, M. Broyer, N. Del Fatti, and F. Vallée Nano Lett., 10, 18531858 (2010).

13. A. Crut, V. Juvé, D. Mongin, P. Maioli, N. Del Fatti, and F. Vallée, Phys. Rev. B. 83, 205430 (2011).

14. H. H. Pan, Z. K. Wang, H. S. Lim, S. C. Ng, V. L. Zhang, M. H. Kuok, T. T. Tran, and X. M. Lu Appl. Phys. Lett. 98133123 (2011).

15. J. Y. Sun, Z. K. Wang, H. S. Lim, S. C. Ng, M. H. Kuok, T. T. Tran, X. Lu, ACSNano, 4, 7692 (2010).

16. Y. Guillet C. Rossignol, B. Audoin, G. Calbris, S. Ravaine, Appl. Phys. Lett. 95, 061909 (2009). 
17. T. Still, M. Mattarelli, D. Kiefer, G. Fytas, M. Montagna, J. Phys. Chem. Lett. 1, 2440 (2010).

18. G. Gantzounis, N. Papanikolaou, N. Stefanou, Phys. Rev. B 84104303 (2011).

19. J. D. Jackson, Classical Electrodynamics, 3rd ed. (Wiley, New York, 1998).

20. N. Stefanou, C. Tserkezis, G. Gantzounis, Proc. SPIE 6989, 698910 (2008).

21. E.D. Palik, Handbook of Optical Constants, 2nd ed. (Academic Press, London, 1998). 